PROCEEDINGS OF THE

AMERICAN MATHEMATICAL SOCIETY

Volume 128, Number 10, Pages 2835-2842

S 0002-9939(00)05464-2

Article electronically published on March 2, 2000

\title{
SEMIGROUPS AND WEIGHTS FOR GROUP REPRESENTATIONS
}

\author{
MOHAN S. PUTCHA
}

(Communicated by Ronald M. Solomon)

\begin{abstract}
Let $G$ be a finite group. Consider a pair $\chi=\left(\chi_{+}, \chi_{-}\right)$of linear characters of subgroups $P, P^{-}$of $G$ with $\chi_{+}$and $\chi_{-}$agreeing on $P \cap P^{-}$. Naturally associated with $\chi$ is a finite monoid $M_{\chi}$. Semigroup representation theory then yields a representation $\theta$ of $G$. If $\theta$ is irreducible, we say that $\chi$ is a weight for $\theta$. When the underlying field is the field of complex numbers, we obtain a formula for the character of $\theta$ in terms of $\chi_{+}$and $\chi_{-}$. We go on to construct weights for some familiar group representations.
\end{abstract}

\section{INTRODUCTION}

A basic theme of representation theory is the construction of an irreducible representation from a linear character (degree 1 representation). In particular, this has been accomplished for finite Lie type groups in the defining characteristic by Curtis, Steinberg and Richen; cf. [5]. Reinterpreting these results, Alperin [1] came up with his famous weight conjectures for irreducible modular representations of arbitrary finite groups.

Motivated by semigroup representation theory [3, Chapter 5], we proceed in this paper in a completely different way. For a finite monoid $M$, the irreducible representations are indexed by the irreducible representations $\theta$ of maximal subgroups $H$ of $M$. We have noted in [13] that $\theta$ gives rise to representations $\theta_{+}, \theta_{-}$(of the same degree) of subgroups $P, P^{-}$of $G$. We observe that even in the situation of irreducible modular representations of a Lie type group [5], a linear character $\theta$ of a Levi subgroup $L$ lifts to linear characters $\theta_{+}$and $\theta_{-}$of associated opposite parabolic subgroups $P, P^{-}$. In this situation there is an associated Lie type monoid [14. In the ordinary representation theory of the symmetric group $S_{n}$ (cf. 6, Section 28]), the irreducible representations are indexed by pairs of linear characters - one trivial and one alternating - of two Young subgroups of $S_{n}$. In this case too, there is a naturally associated monoid. Let $e$ be the primitive idempotent associated with the Young diagram and let $J=S_{n} e S_{n}$. Then

$$
M=S_{n} \cup J \cup\{0\}
$$

is a monoid and all idempotents in $J$ are conjugate, a special property shared by monoids of Lie type.

Received by the editors November 1, 1998.

2000 Mathematics Subject Classification. Primary 20C99, $20 \mathrm{M} 30$.

(C)2000 American Mathematical Society 
Let $G$ be a finite group with a pair of linear characters $\chi=\left(\chi_{+}, \chi_{-}\right)$of subgroups $P, P^{-}$, with $\chi_{+}, \chi_{-}$agreeing on $P \cap P^{-}$. We then construct a monoid $M_{\chi}$, which gives rise to a representation $\theta$ of $G$. When $\theta$ is irreducible, we say that $\chi$ is a weight for $\theta$. Over $\mathbb{C}$, we determine the character of $\theta$ in terms of $\chi_{+}$and $\chi_{-}$. After developing the general theory we construct weights for the Steinberg representation and the unipotent representations of $G L_{n}\left(\mathbb{F}_{q}\right)$.

\section{Semigroups AND Weights}

In this section we introduce our notion of a weight of a group representation, related to semigroup theory. We begin by reviewing some relevant semigroup theory. Let $M$ be a finite monoid with unit group $G$. For $a, b \in M$, Green's relation $\mathcal{J}$ is defined as: $a \mathcal{J} b$ if $M a M=M b M$. For a $\mathcal{J}$-class $J$ of $M$, we can form a semigroup

$$
J^{0}=J \cup\{0\}
$$

where for $a, b \in J$,

$$
a \circ b= \begin{cases}a b & \text { if } a b \in J, \\ 0 & \text { if } a b \notin J\end{cases}
$$

We can then also form the monoid

$$
M(J)=G \cup J^{0} .
$$

Our interest is in the situation when $J^{0}$ is not null, i.e. $J$ has an idempotent $e$. In much of our work, beginning with [10, the following opposite "parabolic" subgroups have played a significant role:

$$
\begin{gathered}
P=P(e)=\{x \in G \mid x e=e x e\}, \\
P^{-}=P^{-}(e)=\{x \in G \mid e x=e x e\} .
\end{gathered}
$$

Let $H=H(e)$ denote the unit group of $e M e$. Then we have homomorphisms $\delta_{+}: P \rightarrow H, \delta_{-}: P^{-} \rightarrow H$, agreeing on $P \cap P^{-}$, given by:

$$
\delta_{+}(p)=p e, \quad \delta_{-}(q)=e q \quad \text { for } p \in P, q \in P^{-} .
$$

The data (1), (2) does not uniquely determine the monoid $M(J)$. However, we have shown ([11, Theorem 1.1], [12 Theorem 1.3]) that if all the idempotents of $J$ are conjugate, then $M(J)$ is uniquely determined by (11), (2). We then denote $M(J)$ by $M\left(G, P, P^{-}, H\right)$, with $\delta_{+}, \delta_{-}$understood to be part of the data.

Let $F$ be an algebraically closed field. By a linear character of $G$, we will mean a representation of degree 1, i.e. a homomorphism into $F^{*}$. Let $\operatorname{Irr} G$ denote the set of irreducible representations of $G$ over $F$. If $\theta \in \operatorname{Irr} G$, we let $\bar{\theta}$ denote the dual representation: $\bar{\theta}(g)=\theta\left(g^{-1}\right)^{t}$. Let $M=M(J)$ and assume that the characteristic of $F$ does not divide $|H|$. Let $F M, F J$ denote the contracted semigroup algebras of $M$ and $J$, respectively. Thus the zero of $J^{0}$ is the zero of $F J$. Clearly $F J$ is an ideal of FM. By semigroup representation theory (3], 4, Chapter 5]),

$$
\begin{gathered}
F J \cong \bigoplus_{\theta \in \operatorname{Irr} H} \mathcal{A}_{\theta}, \\
F J / \operatorname{rad} F J \cong \bigoplus_{\theta \in \operatorname{Irr} H} \mathcal{B}_{\theta}
\end{gathered}
$$

where

$$
\mathcal{B}_{\theta}=\mathcal{A}_{\theta} / \operatorname{rad} \mathcal{A}_{\theta}
$$


is a simple algebra. Here "rad" is the radical. Since $F J / \operatorname{rad} F J$ is an ideal of $F M / \operatorname{rad} F J$, we have irreducible representations $\hat{\theta}: F M \rightarrow \mathcal{B}_{\theta}, \theta \in \operatorname{Irr} H$. These then restrict to representations $\tilde{\theta}$ of $G, \theta \in \operatorname{Irr} H$. We refer to [13] for details. Of particular importance to us is the situation when $\theta$ is a linear character. Then we have linear characters $\chi_{+}=\theta \circ \delta_{+}$and $\chi_{-}=\theta \circ \delta_{-}$of $P$ and $P^{-}$, that agree on $P \cap P^{-}$.

We now reverse the above analysis. Let $P, P^{-}$be subgroups of a finite group $G$. Let $\chi_{+}, \chi_{-}$be linear characters of $P$ and $P^{-}$that agree on $P \cap P^{-}$. Let $\chi=\left(\chi_{+}, \chi_{-}\right)$. Now the subgroup $H$ of $F^{*}$ generated by $\chi_{+}(P)$ and $\chi_{-}\left(P^{-}\right)$is a finite group of order not divisible by the characteristic of $F$. We can therefore form the monoid $M_{\chi}=M(J)=M\left(G, P, P^{-}, H\right)$; cf. [11, Theorem 1.1]. Let $\pi: H \rightarrow F^{*}$ denote the identity map. As in (3), let

$$
\begin{aligned}
& \mathcal{A}_{\chi}=\mathcal{A}_{\pi}, \quad \mathcal{B}_{\chi}=\mathcal{B}_{\pi}, \\
& \hat{\mathcal{A}}_{\chi}=F G+\mathcal{A}_{\chi} .
\end{aligned}
$$

Then $\hat{\mathcal{A}}_{\chi}$ is the algebra over $F$ generated by $G$ and an idempotent $e$, subject to the relations:

$$
\begin{gathered}
p e=\chi_{+}(p) \cdot e, \quad e q=\chi_{-}(q) \cdot e \quad \text { for } p \in P, q \in P^{-}, \\
e g e=0 \quad \text { if } g \in G, g \notin P^{-} P .
\end{gathered}
$$

$\mathcal{A}_{\chi}$ is the span of $G e G$ and is an ideal of $\hat{\mathcal{A}}_{\chi}$. It will be convenient for us to view $\chi$ also as a function with support $P^{-} P$ :

$$
\begin{aligned}
\chi(q p) & =\chi_{-}(q) \chi_{+}(p) \quad \text { if } p \in P, q \in P^{-}, \\
\chi(g) & =0 \quad \text { if } g \in G \backslash P^{-} P .
\end{aligned}
$$

Let the right cosets of $P^{-}$and left cosets of $P$ be, respectively,

$$
\begin{gathered}
P^{-} a_{1}, \ldots, P^{-} a_{m}, \\
b_{1} P, \ldots, b_{n} P .
\end{gathered}
$$

Then $\mathcal{A}_{\chi}$ is a Munn algebra in the sense of [4, Section 5.2] with sandwich matrix,

$$
\Delta=\Delta_{\chi}=\left(\chi\left(a_{i} b_{j}\right)\right) .
$$

We have the representation $\tilde{\pi}: G \rightarrow \mathcal{B}_{\chi}$. If $\tilde{\pi}$ is irreducible, then we say that $\chi=\left(\chi_{+}, \chi_{-}\right)$is a weight (for $\left.\tilde{\pi}\right)$ and define

$$
[\chi]=\left[\chi_{+}, \chi_{-}\right]=\tilde{\pi} .
$$

Let $\theta: G \rightarrow G L(n, F)$ be an irreducible representation. Let $M_{n}(F)$ denote the algebra of all $n \times n$ matrices over $F$. Let $\epsilon$ be a primitive idempotent in $M_{n}(F)$. We may assume that $\epsilon=\left[\begin{array}{ll}1 & 0 \\ 0 & 0\end{array}\right]$. Let $g \in G$. Then

$$
A=\theta(g)=\left[\begin{array}{ll}
a & b \\
C & D
\end{array}\right], \quad \epsilon A \epsilon=\left[\begin{array}{ll}
a & 0 \\
0 & 0
\end{array}\right] .
$$

If $a \neq 0$, then $A$ has an $L U$-decomposition:

$$
A=L U, \quad L=\left[\begin{array}{cc}
c & 0 \\
X & Y
\end{array}\right], U=\left[\begin{array}{cc}
d & Z \\
0 & W
\end{array}\right] .
$$

We will say that $G$ has $L U$-decomposition with respect to $\epsilon$ if $L, U$ can be chosen to be in $\theta(G)$ for all $g \in G$ with $\epsilon \theta(g) \epsilon \neq 0$. 
Theorem 2.1. Let $\theta: G \rightarrow G L(n, F)$ be an irreducible representation. Then

(i) $\theta$ has a weight if and only if $G$ has $L U$-decomposition with respect to some primitive idempotent $\epsilon$ of $M_{n}(F)$.

(ii) $\theta=\left[\chi_{+}, \chi_{-}\right]$with $\chi$ as in (9), if and only if for some primitive idempotent $\epsilon$ of $M_{n}(F)$, and all $p \in P, q \in P^{-}, g \in G \backslash P^{-} P, \theta(p) \epsilon=\chi_{+}(p) \epsilon, \epsilon \theta(q)=\chi_{-}(q) \epsilon$ and $\epsilon \theta(g) \epsilon=0$.

Proof. First we prove (ii). Suppose $\theta=\left[\chi_{+}, \chi_{-}\right]$. Then $\mathcal{B}_{\chi} \cong M_{n}(F)$. By (5), the conditions are satisfied with $\epsilon$ being the image of $e$. Conversely if the conditions are satisfied, then by (5), $\theta$ extends to a homomorphism from $\hat{\mathcal{A}}_{\chi}$ to $M_{n}(F)$ by sending $e$ to $\epsilon$. It follows that $\mathcal{B}_{\chi} \cong M_{n}(F)$ and that $\theta=[\chi]$.

We now prove (i). Suppose $G$ has $L U$-decomposition with respect to a primitive idempotent $\epsilon$ of $M_{n}(F)$. Let

$$
\begin{aligned}
P & =\{x \in G \mid \theta(x) \epsilon=\epsilon \theta(x) \epsilon\}, \\
P^{-} & =\{x \in G \mid \theta(x) \epsilon=\epsilon \theta(x)=\epsilon \theta(x) \epsilon\} .
\end{aligned}
$$

Let $p \in P$. Since $\epsilon$ is primitive, $\theta(p) \epsilon=\chi_{+}(p) \epsilon$ for some $\chi_{+}(p) \in F^{*}$. Similarly for $q \in P^{-}, \epsilon \theta(q)=\chi_{-}(q) \epsilon$ for some $\chi_{-}(q) \in F^{*}$. Then clearly $\chi_{+}, \chi_{-}$are linear characters of $P$ and $P^{-}$, agreeing on $P \cap P^{-}$. If $g \in G$, then by definition, $\epsilon \theta(g) \epsilon \neq 0$ implies that $g \in P^{-} P$. By (ii), $\chi=\left(\chi_{+}, \chi_{-}\right)$is a weight for $\theta$. Conversely, if $\theta=\left[\chi_{+}, \chi_{-}\right]$, then by (ii), $G$ has $L U$-decomposition with respect to some primitive idempotent $\epsilon$ of $M_{n}(F)$.

Example 2.2. Let $G$ be a finite Lie type group defined over $\mathbb{F}_{q}$. Let $S$ denote the set of simple reflections. For $I \subseteq S$, let $P_{I}, P_{I}^{-}, L_{I}$ denote the associated opposite parabolic subgroups and Levi subgroup, respectively. By [5], there is a 1-1 correspondence between the irreducible representations of $G$ over $F=\overline{\mathbb{F}}_{q}$ and pairs $(I, \lambda)$, where $I \subseteq S$, and $\lambda$ is a linear character of $L_{I}$. If a representation $\theta$ of $G$ corresponds to $(I, \lambda)$, let $\chi_{+}=\lambda \circ \delta_{+}, \chi_{-}=\lambda \circ \delta_{-}$, where $\delta_{+}: P_{I} \rightarrow L_{I}, \delta_{-}: P_{I}^{-} \rightarrow$ $L_{I}$ are the natural homomorphisms. Then by [14] and Theorem 2.1, $\theta$ has weight $\left(\chi_{+}, \chi_{-}\right)$. In this case $\left(\chi_{-}, \chi_{+}\right)$is also a weight, but in general $\left[\chi_{-}, \chi_{+}\right] \neq\left[\chi_{+}, \chi_{-}\right]$. This is because $P_{I}$ need not be conjugate to $P_{I}^{-}$. Contrast this with Theorem 3.1 .

Theorem 2.3. Suppose $\chi=\left(\chi_{+}, \chi_{-}\right)$is a weight. Then

(i) $\bar{\chi}=\left(\bar{\chi}_{-}, \bar{\chi}_{+}\right)$is also a weight and $[\bar{\chi}]=\overline{[\chi]}$.

(ii) The degree of $[\chi]$ is equal to the rank of the matrix $\Delta_{\chi}$ of (8).

Proof. $\hat{\mathcal{A}}_{\chi}$ is given by the relations in (5). $\hat{\mathcal{A}}_{\bar{\chi}}$ is the algebra generated by $G$ and an idempotent $\bar{e}$, subject to the relations:

$$
\begin{gathered}
q \bar{e}=\bar{\chi}_{-}(q) \bar{e}, \quad \bar{e} p=\bar{\chi}_{+}(p) \bar{e} \quad \text { for } p \in P, q \in P^{-}, \\
\bar{e} g \bar{e}=0 \quad \text { for } g \in G, g \notin P P^{-} .
\end{gathered}
$$

The map sending $e$ to $\bar{e}$ and $g$ to $g^{-1}, g \in G$, yields an anti-isomorphism between $\hat{\mathcal{A}}_{\chi}$ and $\hat{\mathcal{A}}_{\bar{\chi}}$. This yields a natural anti-isomorphism between $\mathcal{B}_{\chi}$ and $\mathcal{B}_{\bar{\chi}}$. It follows that $\bar{\chi}$ is a weight and that $[\bar{\chi}]$ is the dual of $[\chi]$.

By 3] or [4, Chapter 5], the degree of $[\chi]$ is equal to the rank of the sandwich matrix which is given in (8) . This proves (ii). 


\section{Complex Representations}

In this section we will let $F=\mathbb{C}$. If $\varphi, \psi$ are characters of $G$, then the intertwining number is

$$
\langle\varphi, \psi\rangle=\frac{1}{|G|} \sum_{g \in G} \varphi(g) \overline{\psi(g)} .
$$

If $\varphi$ is a character of a subgroup $P$ of $G$, then the induced character is

$$
\varphi \uparrow G(g)=\frac{1}{|P|} \sum_{\substack{x \in G \\ x g x^{-1} \in P}} \varphi\left(x g x^{-1}\right) .
$$

Theorem 3.1. Let $\chi=\left(\chi_{+}, \chi_{-}\right)$be a weight. Then

(i) $\left(\chi_{-}, \chi_{+}\right)$is also a weight and $[\chi]=\left[\chi_{-}, \chi_{+}\right]$.

(ii) Let $[\chi]$ have degree $m$. Then with the notation (6), the character $\xi$ of $[\chi]$ is given by:

$$
\xi(g)=\frac{m}{|G|} \sum_{x \in G} \chi\left(x g x^{-1}\right) .
$$

Proof. (i) We have an anti-automorphism of $\mathbb{C} G$ given by:

$$
\left(\Sigma \alpha_{g} g\right)^{*}=\Sigma \bar{\alpha}_{g} g^{-1} .
$$

This anti-automorphism fixes the central idempotents and hence the blocks of $\mathbb{C} G$. By Theorem 2.1 $G$ has $L U$-decomposition relative to a primitive idempotent $\epsilon$ in the block of $[\chi]$ such that

$$
\begin{gathered}
p \epsilon=\chi_{+}(p) \epsilon, \quad \epsilon q=\chi_{-}(q) \epsilon, \quad p \in P, q \in P^{-}, \\
\epsilon g \epsilon=0 \quad \text { if } g \in G \backslash P^{-} P .
\end{gathered}
$$

Then $\epsilon^{*}$ is also a primitive idempotent in the block of $[\chi]$ and

$$
\begin{gathered}
\epsilon^{*} p=\chi_{+}(p) \epsilon^{*}, \quad q \epsilon^{*}=\chi_{-}(q) \epsilon^{*}, \quad p \in P, q \in P^{-}, \\
\epsilon^{*} g \epsilon^{*}=0 \text { if } g \notin P P^{-} .
\end{gathered}
$$

By Theorem 2.1, $\left(\chi_{-}, \chi_{+}\right)$is also a weight for $[\chi]$.

(ii) Now $\mathbb{C} G$ is a semisimple algebra with

$$
\mathbb{C} G \cong \bigoplus_{\theta \in \operatorname{Irr} G} \mathcal{C}_{\theta}
$$

where the simple algebra $\mathcal{C}_{\theta}$ is the block of $\theta$. The identity element of $\mathcal{C}_{\theta}$ is:

$$
\eta_{\theta}=\frac{1}{|G|} \sum_{x \in G} \overline{\theta(x)} x .
$$

Let $\pi=[\chi]$ and let $\gamma: G \rightarrow \mathbb{C}$ be defined by

$$
\gamma(g)=\sum_{x \in G} \chi\left(x g x^{-1}\right)
$$


where $\chi(g)$ is as in (6). Then clearly $\gamma$ is a class function of $G$. By Theorem 2.1] $\mathcal{C}_{\pi}$ has a primitive idempotent $\epsilon$ satisfying (12). Then for $\theta \in \operatorname{Irr} G, \theta \neq \pi$,

$$
\begin{aligned}
0 & =\epsilon \eta_{\theta} \quad(\text { by }(13)) \\
& =\epsilon \eta_{\theta} \epsilon \\
& =\frac{1}{|G|} \sum_{g \in G} \overline{\theta(g)} \chi(g) \epsilon \quad(\text { by }(6),(12),(14)) .
\end{aligned}
$$

Since $\theta$ is a class function we see that

$$
\sum_{g \in G} \overline{\theta(g)} \chi\left(x g x^{-1}\right)=0, \quad x \in G .
$$

Summing over all $x \in G$, we see that $\langle\theta, \gamma\rangle=0$. It follows that $\gamma$ is a scalar multiple of $\pi$. Since $\gamma(1)=|G|$, we see that $\pi=\frac{m}{|G|} \gamma=\xi$. This completes the proof.

We now obtain a sufficient condition for $\chi=\left(\chi_{+}, \chi_{-}\right)$to be a weight. Let

$$
\epsilon_{\chi}=\sum_{g \in G} \chi(g) g^{-1}
$$

Theorem 3.2. Suppose $\epsilon_{\chi}$ has rank 1 , i.e. $\operatorname{dim} \epsilon_{\chi} \mathbb{C} G \epsilon_{\chi}=1$. Then $\chi$ is a weight and $\epsilon=\frac{\operatorname{deg}[\chi]}{|G|} \epsilon_{\chi}$ is a primitive idempotent of $\mathbb{C} G$. The corresponding representation of $\mathcal{A}_{\chi}$ is obtained by sending e to $\epsilon$.

Proof. Let

$$
\epsilon_{1}=\frac{1}{|P|} \sum_{p \in P} \chi_{+}(p) p^{-1}, \quad \epsilon_{2}=\frac{1}{|P|} \sum_{q \in P^{-}} \chi_{-}(q) q^{-1}
$$

Then

$$
\epsilon_{1}^{2}=\epsilon_{1}, \quad \epsilon_{2}^{2}=\epsilon_{2}, \quad \epsilon_{1} \epsilon_{2}=\frac{\left|P \cap P^{-}\right|}{|P|\left|P^{-}\right|} \epsilon_{\chi}
$$

By (11),

$$
\epsilon_{2} \epsilon_{1}=\epsilon_{2}^{*} \epsilon_{1}^{*}=\left(\epsilon_{1} \epsilon_{2}\right)^{*}
$$

So by (14), $\epsilon_{1} \epsilon_{2}, \epsilon_{2} \epsilon_{1}$ are rank 1 elements of $\mathcal{C}_{\pi}$ for some $\pi \in \operatorname{Irr} G$. Since $\mathcal{C}_{\pi}$ is a simple algebra, the rank 1 elements of $\mathcal{C}_{\pi}$ form a $\mathcal{J}$-class of the multiplicative semigroup of $\mathcal{C}_{\pi}$. So by [4, Chapter 3], if $a, b, c$ are elements of rank 1 in $\mathbb{C}_{\pi}$, then

$$
a b, b c \neq 0 \Rightarrow a b c \neq 0 .
$$

Let $p \in P, q_{1}, q_{2} \in P^{-}$. Then since $\chi_{+}, \chi_{-}$agree on $P \cap P^{-}$,

$$
q_{1} p q_{2}=1 \Rightarrow p=q_{1}^{-1} q_{2}^{-1} \in P \cap P^{-} \Rightarrow \chi_{-}\left(q_{1}\right) \chi_{+}(p) \chi_{-}\left(q_{2}\right)=1 .
$$

It follows that the coefficient of 1 in $\epsilon_{2} \epsilon_{1} \epsilon_{2}$ is non-zero. Hence $\epsilon_{2} \epsilon_{1} \epsilon_{2} \neq 0$. Similarly $\epsilon_{1} \epsilon_{2} \epsilon_{1} \neq 0$. So by (18),

$$
\left(\epsilon_{1} \epsilon_{2}\right)^{2}=\left(\epsilon_{1} \epsilon_{2}\right)\left(\epsilon_{2} \epsilon_{1}\right)\left(\epsilon_{1} \epsilon_{2}\right) \neq 0 .
$$

By (17), $\epsilon_{\chi}^{2} \neq 0$. Since $\epsilon_{\chi}$ has rank $1, \epsilon_{\chi}^{2}=\alpha \epsilon_{\chi}$ for some $\alpha \in \mathbb{C}, \alpha \neq 0$. So $\epsilon=\frac{1}{\alpha} \epsilon_{\chi}$ is a primitive idempotent of $\mathcal{C}_{\pi}$ The linear operator $E: \mathbb{C} G \rightarrow \mathbb{C} G$ given by

$$
E(x)=\epsilon x, \quad x \in \mathbb{C} G,
$$


is idempotent of rank $\operatorname{deg} \pi$. By (15), $E$ has trace $\frac{|G|}{\alpha}$. Hence $\alpha=\frac{|G|}{\operatorname{det} \pi}$. By (16), (17),

$$
p \epsilon=\chi_{+}(p) \epsilon, \quad e q=\chi_{-}(q) \epsilon \quad \text { for } p \in P, q \in P^{-} .
$$

Let $x \in G, p_{1}, p_{2} \in P, q_{1}, q_{2} \in P^{-}$. Then

$$
p_{1} q_{1} x p_{2} q_{2}=1 \Rightarrow x^{-1}=p_{2} q_{2} p_{1} q_{1} .
$$

Hence the coefficient of 1 in $\epsilon x \epsilon$ is equal to the coefficient of $x^{-1}$ in $\epsilon^{2}=\epsilon$. Since $\epsilon x \epsilon$ is a scalar multiple of $\epsilon$, we see by (15) that

$$
\epsilon x \epsilon \neq 0 \Rightarrow x \in P^{-} P \text {. }
$$

By (19), (20) and Theorem 2.1, $\chi$ is a weight and $[\chi]=\pi$. This completes the proof.

Corollary 3.3. If $\left\langle\chi_{+} \uparrow G, \chi_{-} \uparrow G\right\rangle=1$, then $\chi=\left(\chi_{+}, \chi_{-}\right)$is a weight of the representation corresponding to the common component of $\chi_{+} \uparrow G$ and $\chi_{-} \uparrow G$.

Proof. Let $\pi$ be the common component of $\chi_{+} \uparrow G$ and $\chi_{-} \uparrow G$. Let $\mathcal{C}_{\pi}, \eta_{\pi}$ be as in (13), (14), respectively. Since $\left\langle\chi_{+} \uparrow G, \pi\right\rangle=1=\left\langle\chi_{-} \uparrow G, \pi\right\rangle$, we see by Frobenius reciprocity that $\eta_{\pi} \epsilon_{1}$ and $\eta_{\pi} \epsilon_{2}$ are primitive idempotents and that $\eta_{\theta} \epsilon_{1} \epsilon_{2}=0$ if $\theta \in \operatorname{Irr} G, \theta \neq \pi$. Hence

$$
\epsilon_{1} \epsilon_{2}=\eta_{\pi} \epsilon_{1} \epsilon_{2}=\left(\eta_{\pi} \epsilon_{1}\right)\left(\eta_{\pi} \epsilon_{2}\right)
$$

is a rank 1 element of $\mathcal{C}_{\pi}$. Hence by (17), $\epsilon_{\chi}$ has rank 1. By Theorem 3.2, $\chi$ is a weight.

Example 3.4. Let $G=S_{n}$, the symmetric group of degree $n$. Let $\alpha, \alpha^{\prime}$ be dual partitions of $n$, and $[\alpha]$ the associated irreducible representation of $S_{n}$. Let $\mathcal{S}_{\alpha}, S_{\alpha^{\prime}}$ be the associated Young subgroups with $S_{\alpha} \cap S_{\alpha^{\prime}}=1$. Let $\chi_{+}$be the trivial character on $S_{\alpha}$ and let $\chi_{-}$be the alternating character of $S_{\alpha}$. By [8. Theorem 2.1.3], $\left\langle\chi_{+} \uparrow G, \chi_{-} \uparrow G\right\rangle=1$ with the character of $[\alpha]$ being the common component. Hence by Corollary [3.3, $\left(\chi_{+}, \chi_{-}\right)$is a weight for $[\alpha]$. We also note that $\epsilon_{\chi}$ as in (15) is the associated Young Symmetrizer; cf. [6, Section 2], [7, Section 4.1].

Example 3.5. Let $G$ be a finite Lie type group with opposite Borel subgroups $B, B^{-}$. Let $U$ denote the unipotent radical of $B$. Let $U^{\prime}$ denote the product of the positive root subgroups not corresponding to the simple roots. Let $\chi_{+}$be a linear character of $U$ lifted from a non-degenerate linear character of $U / U^{\prime}$, as in [2] Chapter 8]. So $\chi_{+} \uparrow G$ is the Gel'fand-Graev character of $G$. Hence the irreducible components of $\chi_{+} \uparrow G$ have multiplicity one and are the regular characters of $G$. Let $\chi_{-}$be the trivial character of $B^{-}$. Then the Steinberg character occurs with multiplicity one in $\chi_{-} \uparrow G$ and the irreducible components of $\chi_{-} \uparrow G$ are among the unipotent characters of $G$. By [2, Chapter 12], the Steinberg character is the only character of $G$ that is both regular and unipotent. Hence $\left\langle\chi_{+} \uparrow G, \chi_{-} \uparrow G\right\rangle=1$ with the Steinberg character being the common component. By Corollary 3.3. $\left(\chi_{+}, \chi_{-}\right)$is a weight for the Steinberg representation of $G$.

Example 3.6. Let $G=G L_{n}\left(\mathbb{F}_{q}\right)$. We can combine Examples 3.4 and 3.5 to obtain weights for all the unipotent representations of $G$. Let $\alpha, \alpha^{\prime}$ be dual partitions of $n$ and let $S_{\alpha}, S_{\alpha^{\prime}}$ be associated Young subgroups with $S_{\alpha} \cap S_{\alpha^{\prime}}=1$. Let $P_{\alpha}, P_{\alpha^{\prime}}$ be parabolic subgroups with Weyl groups $S_{\alpha}, S_{\alpha^{\prime}}$, respectively. We may assume that $P_{\alpha}$ consists of block upper triangular matrices. Let $L_{\alpha}$ denote the Levi subgroup 
of block diagonal matrices of $P_{\alpha}$. Let $U$ denote the group of unipotent upper triangular matrices. Let $U^{\prime}$ denote the normal subgroup of $U$ generated by root subgroups with the root not corresponding to a simple reflection of $S_{\alpha}$. Let $\chi_{+}$be a linear character of $U$ obtained by lifting a non-degenerate linear character of the abelian group $U / U^{\prime}$ to $U$. Thus $\chi_{+} \uparrow P_{\alpha}$ is equal to the Gel'fand-Graev character of $L_{\alpha}$ lifted to $P_{\alpha}$.

Since $S_{\alpha} \cap S_{\alpha^{\prime}}=1, P_{\alpha^{\prime}} \cap L_{\alpha}$ is a Borel subgroup of $L_{\alpha}$. Hence, for some $\sigma \in S_{\alpha}$, $\sigma^{-1}\left(P_{\alpha^{\prime}} \cap L_{\alpha}\right) \sigma$ consists of lower triangular matrices. Let $P_{\alpha}^{-}=\sigma^{-1} P_{\alpha^{\prime}} \sigma$ and let $\chi_{-}$be the trivial character on $P_{\alpha}^{-}$. Then

$$
\left.\chi_{+}\right|_{U \cap P_{\alpha}^{-}}=\left.\chi_{-}\right|_{U \cap P_{\alpha}^{-}}, \quad\left\langle\chi_{+} \uparrow G, \chi_{-} \uparrow G\right\rangle=1 .
$$

By Corollary 3.3, $\chi_{\alpha}=\left(\chi_{+}, \chi_{-}\right)$is a weight. Thus $\left[\chi_{\alpha}\right], \alpha$ a partition of $n$, are all the unipotent representations of $G$.

Let $G$ be a finite group of Lie type. The unipotent characters of $G$ have been studied in detail by Lusztig [9]. Finding weights for these unipotent representations remains an open problem.

\section{REFERENCES}

1. J. L. Alperin, Weights for finite groups, Proc. Symp. Pure Math. 47 (1987), 369-379. MR 89h:20015

2. R. W. Carter, Finite groups of Lie type: Conjugacy classes and complex character, Wiley (1985). MR 87d:20060

3. A. H. Clifford, Matrix representations of completely simple semigroups, Amer. J. Math. 64 (1942), 327-342. MR 4:4a

4. A. H. Clifford and G. B. Preston, Algebraic theory of semigroups, Vol. 1, AMS Surveys No. 7, 1961.

5. C. W. Curtis, Modular representations of finite groups with split $(B, N)$-pari, Lecture Notes in Math. 131 (1970), 57-95. MR 41:6991

6. C. W. Curtis and I. Reiner, Representation theory of finite groups and associative algebras, Wiley (1962). MR 26:2519

7. W. Fulton and J. Harris, Representation theory, Graduate Texts in Mathematics 129, Springer-Verlag, 1991. MR 93a:20069

8. G. James and A. Kerber, The representation theory of the symmetric group, Encyclopedia of Mathematics and its applications, Vol. 16, Addison-Wesley, 1981. MR 83k:20003

9. G. Lusztig, Characters of reductive groups over a finite field, Annals. of Math. Studies 107 (1984), Princeton University Press. MR 86j:20038

10. M. S. Putcha, A semigroup approach to linear algebraic groups, J. Algebra 80 (1983), 164-185. MR 84j:20045

11. Wandwich matrices, Solomon algebras and Kazhdan-Lusztig polynomials, Trans. Amer. Math. Soc. 340 (1993), 415-428. MR 94a:20112

12. _ Classification of monoids of Lie type, J. Algebra 163 (1994), 636-662. MR 95b:20089

13. Complex representations of finite monoids, Proc. London Math. Soc. 73 (1996), 623-641. MR 97e:20093

14. M. S. Putcha and L. E. Renner, The canonical compactification of a finite group of Lie type, Trans. Amer. Math. Soc. 337 (1993), 305-319. MR 93g:20123

Department of Mathematics, North Carolina State University, Raleigh, North CarOLINA 27695-8205

E-mail address: putcha@math.ncsu.edu 